\title{
Amplitude and polarization transformations of the Bessel beam as it passes through an anisotropic crystal perpendicular to the axis of the crystal
}

\author{
A.V. Glazkova ${ }^{1}$, M.V. Zablovskaya ${ }^{1}$, V.V. Podlipnov ${ }^{1,2}$ \\ ${ }^{1}$ Samara National Research University, 34 Moskovskoe Shosse, 443086, Samara, Russia \\ ${ }^{2}$ Image Processing Systems Institute - Branch of the Federal Scientific Research Centre "Crystallography and Photonics" of Russian Academy of Sciences, 151 \\ Molodogvardeyskaya st., 443001, Samara, Russia
}

\begin{abstract}
A comparative numerical calculation of the propagation of a zero-order Bessel laser beam in a uniaxial crystal perpendicular to its axis is performed using the Rayleigh-Sommerfeld integral operator, generalized for an anisotropic environment. Numerical simulation is performed with a different type of beam polarization and different characteristics of the Bessel beam. Patterns of the beam intensity during the passing of different distances in the crystal are obtained, showing the degree of astigmatic transformation, which makes it possible to determine the conditions under which the greatest astigmatic distortion of the beams occurs. The above analysis can be useful in practice for determining the anisotropy characteristics of a crystal.
\end{abstract}

Keywords: diffraction axicon; birefringent crystal; polarization transformations; amplitude transformations, Bessel beams; astigmatism

\section{Introduction}

Optical devices are becoming more and more interesting and practical. They allow to transform certain properties of electromagnetic radiation into others. Most often, modal transformations (from the fundamental mode to higher order distributions) and polarization (from homogeneous linear polarization to more complex ones) are required. One of the tools of such transformations are anisotropic crystals. The propagation of laser modes with a high numerical aperture in an environment with strong anisotropy leads to complex polarization-mode transformations [1-6].

In particular, when propagating along the crystal axis, the spin angular momentum is transformed, which has a circularly polarized beam at the orbital angular momentum [7-13]. It was shown in [6, 7, 11-13] that when propagating along the crystal axis, nonparaxial Bessel beams undergo a periodic change in intensity, corresponding to a transformation into a higher-order beam. In publications [14-20], polarization transformations of beams focused along the crystal axis were considered.

The propagation of various types of laser beams perpendicular to the axis of the crystal was investigated in [21-26]. The most interesting transformations were observed for Bessel beams [16, 21, 24, 27], since in this case there is a visually pronounced astigmatic distortion of the ring structure of the beam. A similar distortion can be observed with oblique incidence of a plane wave on a diffraction axicon [28-30], and also with a cylindrical lens [31]. This analogy was noted in [24], and the analytical basis for such an effect was given in [27].

In this paper, the effect of the astigmatic transformation of Bessel beams propagating perpendicular to the crystal axis is studied in detail on the basis of numerical simulation. The calculation was carried out using the Rayleigh-Sommerfeld integral operator, generalized for an anisotropic environment [32,33]. Numerical simulation is performed for different types of beam polarization and different characteristics of the Bessel beam. The formation of Bessel beams [34-37] was carried out with the diffraction axicon with different period of the radial lattice. The effect of the relative position of the polarization plane of the radiation and the c-axis of the crystal on the intensity distributions formed in different vector components of ordinary and extraordinary beams is investigated. Patterns of the beam intensity are obtained during the passing of different distances in the crystal, showing the degree of astigmatic transformation, which makes it possible to determine the conditions under which the greatest astigmatic distortion of the beams occurs. The above analysis can be useful in practice for determining the anisotropy characteristics of a crystal.

\section{Theoretical analysis}

Consider an anisotropic crystal whose axis is oriented perpendicular to the propagation axis and coincides with the Oy axis. In this case, the field propagation in a crystal with dielectric permittivities, (ordinary and extraordinary) can be described by an expression similar to the Rayleigh-Sommerfeld integral [32, 33]:

$$
\mathbf{E}(u, v, z)=\frac{2 \pi z}{\lambda^{2}} \sum_{j=1}^{2} \iint \mathbf{e}_{j}\left(\alpha_{j c}, \beta_{j c}\right)\left[\mathbf{w}_{j}\left(\alpha_{j c}, \beta_{j c}\right)^{T} \mathbf{E}_{\perp}(x, y, 0)\right] \frac{\sqrt{d_{j} s_{j} t_{j}}}{R_{j}^{2}} \exp \left\{i k \sqrt{\frac{d_{j}}{s_{j} t_{j}}} R_{j}\right\} \mathrm{d} x \mathrm{~d} y,
$$

where the indices correspond to the ordinary $(\mathrm{j}=1)$ and extraordinary $(\mathrm{j}=2)$ waves, $d_{1}=d_{2}=\varepsilon_{o}, s_{1}=t_{1}=1, s_{2}=t_{2}=\varepsilon_{o} / \varepsilon_{e}$.

For transverse (x- and $\mathrm{y}$ - components): 


$$
\begin{aligned}
& e_{1 x}(\alpha, \beta)=1, \\
& e_{1 y}(\alpha, \beta)=0, \\
& e_{2 x}(\alpha, \beta)=\alpha \beta, \\
& e_{2 y}(\alpha, \beta)=\beta^{2}-\varepsilon_{o} . \\
& \mathbf{w}_{1}(\alpha, \beta)^{T}=\left(1, \frac{\alpha \beta}{\left(\varepsilon_{o}-\beta^{2}\right)}\right), \\
& \mathbf{w}_{2}(\alpha, \beta)^{T}=\left(0,-\frac{1}{\left(\varepsilon_{o}-\beta^{2}\right)}\right) . \\
& \left\{\begin{array}{l}
\alpha_{1 c}=\sqrt{\varepsilon_{o}} \frac{(u-x)}{R_{1}}, \quad\left\{\begin{array}{l}
\alpha_{1 c}=\sqrt{\varepsilon_{o}} \frac{(u-x)}{R_{1}}, \\
\beta_{1 c}=\sqrt{\varepsilon_{o}} \frac{(v-y)}{R_{1}},
\end{array} \beta_{1 c}=\sqrt{\varepsilon_{o}} \frac{(v-y)}{R_{1}},\right.
\end{array}\right.
\end{aligned}
$$

where

$$
\begin{aligned}
& R_{1}=\sqrt{(u-x)^{2}+(v-y)^{2}+z^{2}}, \\
& R_{2}=\sqrt{\frac{\varepsilon_{e}}{\varepsilon_{o}}} \sqrt{(u-x)^{2}+\frac{\varepsilon_{o}}{\varepsilon_{e}}(v-y)^{2}+z^{2}} .
\end{aligned}
$$

Similar results can be obtained if the crystal axis is directed along the Ox axis.

\section{Results of numerical simulation}

During the experiment, the axicon was used. The scheme of the axicon's work is shown in Fig. 1

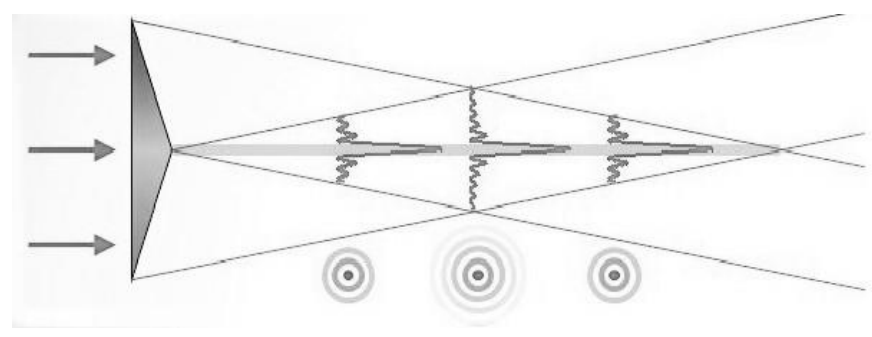

Fig. 1. The scheme of the axicon's work.

In order to carry out the simulation as an anisotropic medium, a lithium niobate crystal of the $\mathrm{X}$-cut was chosen in this study, the dielectric constant of which is $\varepsilon_{0}=5.2273505956, \varepsilon_{\mathrm{e}}=4.8517551289$. The refractive indices of this crystal are: $\mathrm{n}_{0}=$ $2.28634, n_{e}=2.20267$. For the formation of zero-order Bessel beams, diffraction axicons with periods $d_{1}=1.2 \mu \mathrm{m}, d_{2}=2 \mu \mathrm{m}, d_{3}$ $=4 \mu \mathrm{m}$ were used and illuminated with light polarized linearly along the OY axis with a wavelength of $=632.8 \mathrm{~nm}$. We also compared the results of the transformation for different crystal thicknesses, which were chosen $\mathrm{h}_{1}=1047 \mu \mathrm{m}$ and $\mathrm{h}_{2}=843 \mu \mathrm{m}$. To analyze the transformation of Bessel beams with axicons, the results of the simulation were presented in the form of patterns of light distribution of propagating beams separately for polarized light along OX, separately for OY and their superposition. The results of the simulation are presented in Table 1.

It can be noted that the picture of the $\mathrm{Y}$ component almost does not differ from the superposition picture of the $\mathrm{X}$ and $\mathrm{Y}$ components, which means that the $\mathrm{X}$ component has a negligible intensity, and the linearly polarized light at the exit from the lithium niobate crystal has not changed its polarization.

As can be seen from the modeled intensity distribution maps of lithium niobate transformed into an anisotropic lithium crystal by Bessel beams, the beams formed by axicons with the minimal period are subjected to the strongest astigmatic distortions. With an increase in crystal thickness, the degree of astigmatism increases in proportion to the propagation length.

When analyzing patterns of light intensity distribution at the output of an anisotropic crystal for linearly polarized light along the $\mathrm{Y}$ axis, with circular polarization, polarization rotated through an angle of $45^{\circ}$ about the $\mathrm{X}$ axis, the above-described character of the Bessel beam transformation is preserved. 
Computer Optics and Nanophotonics / A.V. Glazkova, M.V. Zablovskaya, V.V. Podlipnov

Table 1. Patterns of propagation of Bessel beams formed with axicons under illumination by light polarized along the OY axis through an anisotropic X-cut

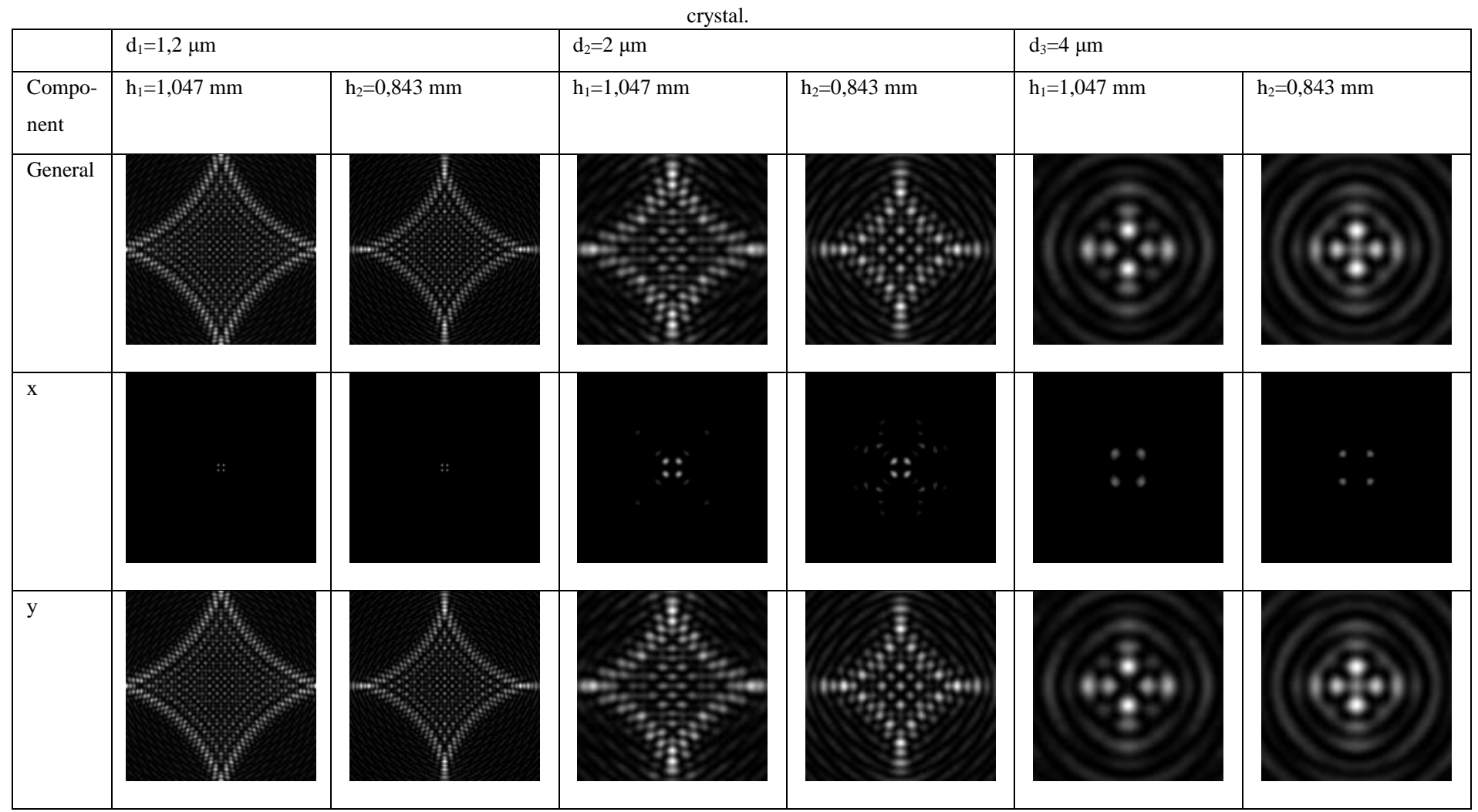

\section{Conclusion}

In the work, to analyze the dependence of the propagation of the zero-order Bessel beam on the polarization angle, on the period and the radius of the axicon, we used the calculation with the Rayleigh-Sommerfeld integral operator generalized for an anisotropic medium. The Bessel beams formed by an axicon with the smallest period and passing through an anisotropic Crystal at the greatest distance. The described regularities can be used in practice to determine the degree of anisotropy or the exact thickness of the crystal cuts.

\section{Acknowledgments}

This work was supported by the Ministry of Education of the Russian Federation., by the Russian Foundation for Basic Research (RFBR grants 16-07-00825, 16-29-11698 ofi_m and 16-07-00494 a) and by the grant from the President of the Russian Federation (project no. MD- 5205.2016.9).

\section{References}

[1] Zhou Y, Wang X, Dai Ch. Nonparaxial analysis in the propagation of a cylindrical vector Laguerre-Gaussian beam in a uniaxial crystal orthogonal to the optical axis. Opt. Commun. 2013; 305: 113-125.

[2] Khonina SN, Volotovsky SG, Kharitonov SI, Features of nonparaxial propagation of Gaussian and Bessel beams along the axis of the crystal. Computer Optics 2013; 37(3): 297-306.

[3] Loussert C, Brasselet E. Efficient scalar and vectorial singular beam shaping using homogeneous anisotropic media. Opt. Lett. 2010; 35(1): 7-9.

[4] Khonina SN, Zoteeva OV, Kharitonov SI. Nonparaxial propagation of gaussian beams on the angle to the axis of the anisotropic crystal. Computer Optics 2012; 36(3): 346-356.

[5] Khonina SN, Zoteeva OV, Kharitonov SI, Sharp focusing of laser beams in anisotropic uniaxial crystals. Journal of Optical Technology 2015; 82(4): 212219.

[6] Khonina SN, Kharitonov SI. Comparative investigation of nonparaxial mode propagation along the axis of uniaxial crystal. J. Mod. Opt. 2015; 62(2): 125134.

[7] Khilo NA, Petrova ES, Ryzhevich AA. Transformation of the order of Bessel beams in uniaxial crystals. Quantum Electronics 2001; 31(1): 85-89.

[8] Ciattoni A, Cincotti G, Palma C. Circularly polarized beams and vortex generation in uniaxial media. J. Opt. Soc. Am. A $2003 ; 20(1): 163-171$.

[9] Marrucci L, Manzo C, Paparo D. Optical spin-to-orbital angular momentum conversion in inhomogeneous anisotropic media. Phys. Rev. Lett 2006; 96: $163905-163908$.

[10] Picon A, Benseny A, Mompart J, Calvo GF. Spin and orbital angular momentum propagation in anisotropic media: theory. J. Opt 2011; 13 : 064019 (7 pp).

[11] Khilo NA. Diffraction and order conversion of Bessel beams in uniaxial crystals. Opt. Commun. 2012; 285(5): 503-509.

[12] Khonina SN, Morozov AA, Karpeev SV. Effective transformation of a zero-order Bessel beam into a second-order vortex beam using a uniaxial crystal. Laser Phys. 2014; 24(5): 056101 (5 pp).

[13] Khonina SN, Karpeev SV, Morozov AA, Paranin VD. Implementation of ordinary and extraordinary beams interference by application of diffractive optical elements. Journal of Modern Optics 2016; 63(13): 1239-1247. 
Computer Optics and Nanophotonics / A.V. Glazkova, M.V. Zablovskaya, V.V. Podlipnov

[14] Machavariani G, Lumer Y, Moshe I, Meir A, Jackel S, Davidson N. Birefringence-induced bifocusing for selection of radially or azimuthally polarized laser modes. Applied Optics 2007; 46: 3304-3310.

[15] Yonezawa K, Kozawa Y, Sato S. Compact laser with radial polarization using birefringent laser medium. Japanese Journal of Applied Physics 2007; 46: 5160-5163.

[16] Hacyan S, Jáuregui R. Evolution of optical phase and polarization vortices in birefringent media. J. Opt. A: Pure Appl. Opt. $2009 ; 11(8)$ : 085204.

[17] Fadeyeva T, Shvedov V, Shostka N, Alexeyev C, Volyar A. Natural shaping of the cylindrically polarized beams. Optics Letters $2010 ; 35(22)$ : $3787-3789$.

[18] Fadeyeva TA, Shvedov VG, Izdebskaya YV, Volyar AV, Brasselet E, Neshev DN, Desyatnikov AS, Krolikowski W, Kivshar YS. Spatially engineered polarization states and optical vortices in uniaxial crystals. Optics Express 2010; 18(10): 10848-10863.

[19] Khonina SN, Karpeev SV, Alferov SV. Theoretical and an experimental research of polarizing transformations in uniaxial crystals for generation cylindrical vector beams of high orders. Computer Optics 2014; 38(2): 171-180.

[20] Khonina SN, Karpeev SV, Alferov SV, Soifer VA. Generation of cylindrical vector beams of high orders using uniaxial crystals. Journal of Optics 2015; 17: 065001 (11 pp).

[21] Ciattoni A, Palma C. Nondiffracting beams in uniaxial media propagating orthogonally to the optical axis. Opt. Commun. 2003 ; $224(4)$ : $175-183$.

[22] Liu D, Zhou Z. Various dark hollow beams propagating in uniaxial crystals orthogonal to the optical axis. J. Opt. A: Pure Appl. Opt. 2008; 10(9): 095005 (9 pp).

[23] Tang B. Hermite-cosine-Gaussian beams propagating in uniaxial crystals orthogonal to the optical axis. J. Opt. Soc. Am. A $2009 ; 26(12)$ : $2480-2487$.

[24] Zusin DH, Maksimenka R, Filippov VV. Bessel beam transformation by anisotropic crystals. J. Opt. Soc. Am. A 2010; 27(8): 1828-1833.

[25] Zhao C, Cai Y. Paraxial propagation of Lorentz and Lorentz-Gauss beams in uniaxial crystals orthogonal to the optical axis. J. Mod. Opt 2010; 57(5): $375-384$.

[26] Zhou G, Chen R, Chu X. Propagation of Airy beams in uniaxial crystals orthogonal to the optical axis. Opt. Express 2012; 20(3): 2196-2205.

[27] Khonina SN, Paranin VD, Ustinov AV, Krasnov AP. Astigmatic transformation of Bessel beams in a uniaxial crystal. Optica Applicata 2016; 46(1): 5-18.

[28] Bin Z, Zhu L. Diffraction property of an axicon in oblique illumination. Appl. Opt. 1998; 37(13): 2563-2568.

[29] Khonina SN, Kotlyar VV, Soifer VA. Astigmatic Bessel laser beams. J. Mod. Opt. 2004; 51(5): $677-686$.

[30] Bendersky A, Quintian FP, Rebollo MA. Modification of the structure of Bessel beams under oblique incidence. J. Mod. Opt. 2008; 55(15): 2449-2456.

[31] Anguiano-Morales M. Transformation of Bessel beams by means of a cylindrical lens. Appl. Opt. 2009; 48(25): 4826-4831.

[32] Khonina SN, Kharitonov SI. Analogue of Rayleigh-Sommerfeld integral for anisotropic and gyrotropic media. Computer Optics 2012 ; 36 (2): 172-182.

[33] Khonina SN, Kharitonov SI. An analog of the Rayleigh-Sommerfeld integral for anisotropic and gyrotropic media. Journal of Modern Optics 2012; 60(10): 814-822.

[34] Turunen J, Vasara A, Friberg AT. Holographic generation of diffraction-free beams. J. Appl. Opt 1988; 27(19): 3959-3962.

[35] Khonina SN, Kotlyar VV. Bessel-mode formers. Proceedings of SPIE 1994; 2363: 184-190.

[36] Kotlyar VV, Khonina SN, Soifer VA. Algorithm for the generation of non-diffracting Bessel modes. Journal of Modern Optics 1995; $42(6)$ : $1231-1239$.

[37] Chattrapiban N, Rogers EA, Cofield D, Hill WT, Roy R. Generation of nondiffracting Bessel beams by use of a spatial light modulator. Opt. Lett 2003; 28(22): 2183-2185. 